\title{
HOMOTOPY PERTURBATION METHOD COMBINED WITH TREFFTZ METHOD IN NUMERICAL IDENTIFICATION OF LIQUID TEMPERATURE IN FLOW BOILING
}

\section{SYlWIA HoŻEJOWSKA}

Kielce University of Technology, Faculty of Management and Computer Modelling, Kielce, Poland

e-mail: ztpsf@tu.kielce.pl

The paper is focused on numerical identification of 2D temperature fields in flow boiling of the liquid through a horizontal minichannel with a rectangular cross-section. The heat transfer process in the minichannel is described by a two-dimensional energy equation with the corresponding boundary conditions. Liquid temperature is determined using the homotopy perturbation method (HPM) with Trefftz functions for Laplace'a equation. The numerical solution to the energy equation found with the HPM is compared with the solution obtained for the simplified form of the energy equation. Considering that only the thermal sublayer is taken into account, both solutions give similar results.

Keywords: homotopy perturbation method, Trefftz method, flow boiling, inverse problem

\section{Nomenclature}

$\begin{array}{lll}a & - & \text { thermal diffusivity }\left[\mathrm{m}^{2} / \mathrm{s}\right] \\ a, b, c, d & - & \text { approximation coefficients } \\ c_{p} & - & \text { specific heat }[\mathrm{J} /(\mathrm{kg} \mathrm{K})] \\ D & - & \text { hydraulic diametrer }[\mathrm{m}] \\ f & - & \text { friction coefficient } \\ G & - & \text { mass flux }\left[\mathrm{kg} /\left(\mathrm{m}^{2} \mathrm{~s}\right)\right] \\ h & - & \text { homotopy } \\ H & - & \text { minichannel length }[\mathrm{m}] \\ N, M & - & \text { number of Trefftz functions } \\ p & - & \text { pressure }[\mathrm{Pa}] \\ \mathrm{Pr} & - & \text { Prandtl number } \\ q & - & \text { heat flux }\left[\mathrm{W} / \mathrm{m}^{2}\right] \\ q_{V} & - & \text { volumetric heat flux }\left[\mathrm{W} / \mathrm{m}^{3}\right] \\ \mathrm{Re} & - & \text { Reynolds number } \\ S & - & \text { section area }\left[\mathrm{m}^{2}\right] \\ T & - & \text { temperature }[\mathrm{K}] \\ u & - & \text { Trefftz function } \\ w & - & \text { velocity }[\mathrm{m} / \mathrm{s}] \\ x & - & \text { distance along minichannel length }[\mathrm{m}] \\ y & - & \text { distance along glass, foil and liquid }[\mathrm{m}] \\ \alpha & - & \text { heat transfer coefficient }\left[\mathrm{W} /\left(\mathrm{m}^{2} \mathrm{~K}\right)\right] \\ \delta & - & \text { thickness, depth }[\mathrm{m}] \\ \varphi & - & \text { void fraction } \\ \lambda & - & \text { thermal conductivity }[\mathrm{W} /(\mathrm{mK})] \\ \mu & - & \text { dynamic viscosity }[\mathrm{Pas}] \\ \rho & - & \text { density }\left[\mathrm{kg} / \mathrm{m}^{3}\right]\end{array}$


Subscripts

ave - average, $G$ - glass, $F$ - foil, $f$ - liquid, $h$ - hydraulic, in - inlet, $k$ - measurement point, $n$ - number of function, $M$ - minichannel, out - outlet, sat - saturation, $T$ - thermal layer, 0 - initial approximation

Superscripts

$n$ - refers to number of function, $\simeq$ - refers to particular solution

\section{Introduction}

The Trefftz method, first described by Trefftz (1926), is used for solving any partial differential equations that are linear. The method involves approximating the unknown solution using a linear combination of functions that exactly satisfy the given differential equation, i.e., Tref$\mathrm{ftz}$ functions. The linear combination coefficients are determined through minimizing the error functional that describes the mean-square error between the approximate solution and the adopted boundary conditions. In Ciałkowski and Frąckowiak (2002), Herrera (2000), Maciąg (2011), Zieliński (1995) the authors reported the use of Trefftz functions for solving direct and inverse problems in mechanics.

The homotopy perturbation method (HPM) proposed by He (1999) is a useful tool for obtaining exact and approximate solutions to linear and nonlinear partial differential equations. The unknown solution to the differential equation is expressed as the summation of an infinite series that is supposed to be convergent to the exact solution. The HPM procedure generally requires few calculation steps to achieve the accuracy of the solutions. Additional information on HPM can be found in He (2000, 2006), Momani and Odibat (2007), Rajabi et al. (2007), Jafari and Seifi (2009), Słota (2011). Application of HPM to the solution of direct and inverse stationary and non stationary heat conduction problems was presented in Hetmaniok et al. (2012), Al-Khatib et al. (2014) and Grysa et al. (2012).

The paper presents a two-dimensional mathematical model describing heat transfer in flow boiling in an asymmetrically heated rectangular minichannel. In each of the three domains of the test section: the glass pane, the heating foil and the liquid, the heat transfer process has been described by different differential equations with appropriate boundary conditions. The solution of these equations leads to the solution of a threefold conjugated heat transfer problem consisting of a direct problem (in the glass pane) and two inverse problems (in the heating foil and boiling liquid). The Trefftz functions for Laplace's equation are used to determine twodimensional temperature distributions in the glass pane, in the heating foil and in the liquid. The aim of this study is to apply the HPM coupled with the Trefftz method to find the twodimensional temperature distribution of the boiling liquid flowing in an asymmetrically heated horizontal minichannel. Known liquid and foil temperature distributions help determination of the heat transfer coefficient from the Robin condition.

\section{Experiment}

Discussed in detail by Piasecka $(2013,2014)$ the experimental approach to this issue is described below in brief. In the experiment in which the difference between temperatures of the heating foil and the liquid is small, heat transfer enhancement occurs through the phase change that accompanies the boiling process. A microstructured heating surface (the heating foil is enhanced on the side of the fluid) additionally intensifies the process which described Piasecka $(2013,2014)$.

The basic module of the experimental stand is the test section with a minichannel and cooling liquid FC-72 flowing through it, seeFig. 1. One of the walls of the minichannel, made of 
the heating foil supplied with the controlled direct current, is isolated with a glass pane from the outside environment. A thin layer of liquid crystals deposited on the exterior of the foil helps measurement of two-dimensional temperature distribution. Boiling liquid flow structures are observed through the glass pane closing the minichannel on the other side of the flow. The measurements included the local temperature of the heating foil, liquid inlet and outlet temperatures and pressure, current and voltage drop of the electric power supplied to the foil, local void fraction and mass flux. Numerical calculations for FC-72 were performed based on the experiment and on the results described in detail in Piasecka (2014).

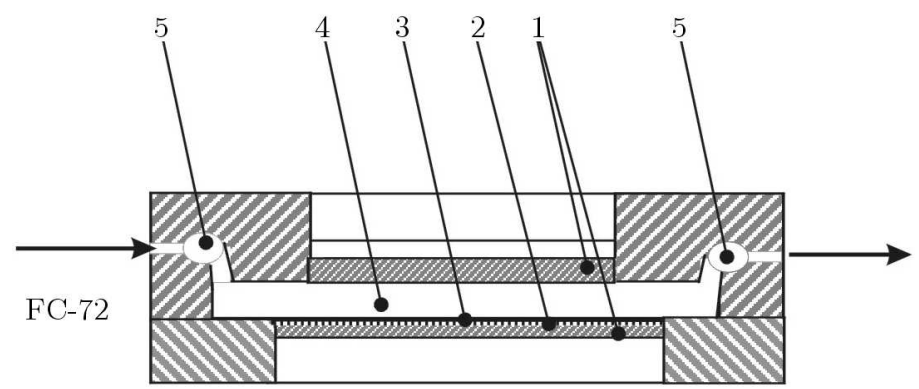

Fig. 1. Measuring module: 1 - glass panes, 2 - heating foil, 3 - thermosensitive liquid crystals, 4 - minichannel, 5 - thermocouples and pressure gauges (pictorial view, not to scale)

\section{Mathematical model}

For simplicity, only two dimensions are taken into account: dimension $x$ along the flow direction and dimension $y$, perpendicular to the flow direction, relating to the thickness of the protecting glass $\left(\delta_{G}\right)$ and the foil $\left(\delta_{F}\right)$, and to the depth of the channel $\left(\delta_{M}\right)$, Fig. 2. We focused on the central part of the measurement module (along its length) so that the physical phenomena on the side edges did not affect the thermodynamic parameters within the investigated segment, Fig. 2. The fluid flow in the minichannel was assumed to be steady, stationary and laminar (Re < 2000) with a constant mass flux, (Hożejowska et al., 2014; Hożejowska and Piasecka, 2014). The velocity vector had only one component $w(y)$ parallel to the heating foil (with other components equal to zero) given by the formula

$$
w(y)=\frac{\Delta p}{2 \mu H}\left(\delta_{M} y-y^{2}\right)
$$

Thus the energy equation exclusively for the liquid phase can be written as follows

$$
L T_{f}=A T_{f}
$$

where $L=\partial^{2} / \partial x^{2}+\partial^{2} / \partial y^{2}$ is the Laplace operator and $A$ is the differential operator defined as

$$
A=\frac{w(y)}{a} \frac{\partial}{\partial x} \quad a=\frac{\lambda_{f}}{c_{p, f} \rho_{f}}
$$

The boundary conditions for equation (3.2) are as follows (Bohdal, 2000; Hożejowska et al., 2014):

- liquid temperatures at the inlet and outlet of the minichannel are known

$$
\left.\begin{array}{l}
T_{f}(0, y)=T_{\text {in }} \\
T_{f}(H, y)=T_{\text {out }}
\end{array}\right\} \quad \text { for } \quad 0 \leqslant y \leqslant \delta_{M}
$$


— liquid temperature in the domain of contact with the heating foil meets the condition

$$
T_{f}\left(x, \delta_{G}+\delta_{F}\right)=\left\{\begin{array}{lll}
T_{F}(x, 0) & \text { if } & T_{F}(x, 0)<T_{\text {sat }}(x) \\
T_{\text {sat }}(x) & \text { if } & T_{F}(x, 0) \geqslant T_{\text {sat }}(x)
\end{array}\right.
$$

where $T_{\text {sat }}$ is the saturation temperature dependent on the pressure $p(x)$ which changes linearly along the minichannel,

- the two-phase mixture per unit volume in the minichannel contains vapour phase and liquid phase in proportion $\varphi$ and $(1-\varphi)$, respectively. The same proportions of vapour and liquid phases are assumed to refer to any cross-sectional area of the minichannel and then to the heat transfer surface. For bubbly and bubbly-slug flows, following Bohdal (2000), Hożejowska et al. (2014), the whole heat flux generated in the foil is assumed to be transferred to the liquid phase in proportion carried over from the void fraction

$$
\lambda_{f} \frac{\partial T_{f}}{\partial y}=\lambda_{F}(1-\varphi(x)) \frac{\partial T_{F}}{\partial y} \quad \text { for } \quad y=0 \quad \text { and } \quad 0 \leqslant x \leqslant H
$$

Figure 2 shows a diagram of the unit with the minichannel and the boundary conditions.

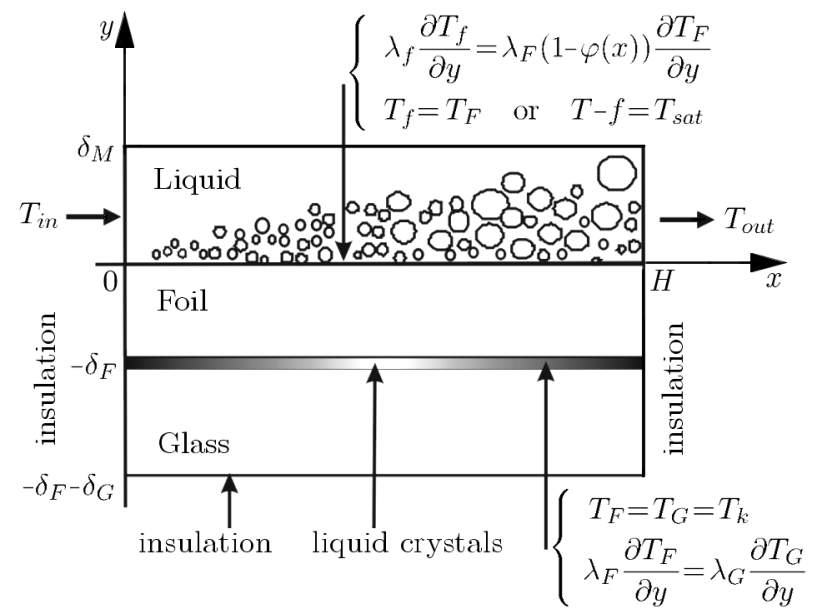

Fig. 2. Scheme of the measuring module with boundary conditions (pictorial view, not to scale)

The temperatures of the heating foil and the glass are assumed to satisfy the following equations (Hożejowska et al., 2009; Piasecka et al., 2004):

— in the glass

$$
L T_{G}=0
$$

— in the foil

$$
L T_{F}=-\frac{q_{V}}{\lambda_{F}}
$$

The conditions at the glass-foil contact can be written as

$$
\begin{aligned}
& T_{F}\left(x_{k},-\delta_{F}\right)=T_{G}\left(x_{k},-\delta_{F}\right)=T_{k} \\
& \lambda_{F} \frac{\partial T_{F}}{\partial y}=\lambda_{G} \frac{\partial T_{G}}{\partial y} \quad y=-\delta_{F} \quad 0 \leqslant x \leqslant H
\end{aligned}
$$

where $T_{k}$ denotes the temperature measured at the glass-foil interface at discrete points $\left(x_{k},-\delta_{F}\right)$ using liquid crystals thermography. The remaining boundaries are assumed to be isolated, Fig. 2 . 
When the heating foil temperature distribution and the temperature gradient are known, the heat transfer coefficient $\alpha(x)$ at the foil-liquid interface can be determined from the Robin condition

$$
-\lambda_{F} \frac{\partial T_{F}}{\partial y}(x, 0)=\alpha(x)\left[T_{F}(x, 0)-T_{f, a v e}(x)\right]
$$

The reference temperature $T_{f, a v e}$ is determined as a mean liquid temperature in the thermal layer

$$
T_{f, \text { ave }}(x)=\frac{1}{\delta_{T}} \int_{0}^{\delta_{T}} T_{f}(x, y) d y
$$

where $\delta_{T}$ is the thickness of the thermal boundary layer determined by Bohdal (2000)

$$
\delta_{T}=\stackrel{-\frac{1}{3}}{\operatorname{Pr}} \delta_{h}
$$

and

$$
\delta_{h}=\frac{2 \mu_{f}}{f w_{\text {ave }} \rho_{f}} \quad f=\frac{64}{\operatorname{Re}} \quad \operatorname{Re}=\frac{w_{\text {ave }} \rho_{f} D}{\mu_{f}}
$$

and $w_{\text {ave }}$ is the mean velocity of the liquid, calculated from

$$
w_{\text {ave }}=\frac{1}{0.5 \delta_{M}} \int_{0}^{0.5 \delta_{M}} w(y) d y
$$

\section{Numerical methods}

\subsection{Trefftz method}

The Trefftz method has been used to calculate approximate two-dimensional temperature distributions of the glass pane and the heating foil. The unknown distributions of $T_{G}$ and $T_{F}$ have been approximated with a linear combination of the Trefftz functions $u_{i}(x, y)$ adequate for Laplace's equation (3.6) (Piasecka et al., 2004), in this case harmonic polynomials, that is

$$
T_{G}(x, y)=\sum_{i=1}^{N_{G}} a_{i} u_{i}(x, y) \quad T_{F}(x, y)=\widetilde{u}(x, y)+\sum_{j=1}^{N_{F}} b_{j} u_{j}(x, y)
$$

where $\widetilde{u}(x, y)$ is the particular solution to equation (3.7). The unknown coefficients $a_{i}$ and $b_{j}$ of linear combinations (4.1) are calculated using the least square method which led to minimizing the functionals suitable for each function $T_{G}$ and $T_{F}$. These functionals describe the mean squared error between the approximates and prescribed boundary conditions. This procedure was thoroughly discussed in Piasecka et al. (2004) and Hożejowska et al. (2009).

Numerical computations have been made sequentially. We obtained the solution first in the glass, and then in the heating foil. The approximate functions $T_{G}$ and $T_{F}$, obtained with the Trefftz method, satisfied exactly equations (3.6) and (3.7), respectively, and approximately the adopted boundary conditions. Solving these equations has led to solving two heat transfer problems: the direct problem in the glass and then the inverse problem in the heating foil. Fluid temperature has been computed in the next stage by solving the inverse problem with the HPM and Trefftz method combined. 


\subsection{Homotopy perturbation method (HPM)}

The use of the HPM in combination with the Trefftz method for identifying the source function was presented in Grysa and Maciąg (2013) and Al-Khatib et al. (2014). In this study, the above combination is used to find the two-dimensional liquid temperature distribution in the minichannel. According to the homotopy method, a homotopy $h(x, y, p)$ can be constructed as the solution to

$$
(1-p)\left[L(h)-L\left(u_{0}\right)\right]+p[L(h)-A(h)]=0
$$

where the parameter $0 \leqslant p \leqslant 1$ and $u_{0}$ is the initial approximation of equation (2) that satisfies boundary conditions (3.3)-(3.5). Substituting $p=1$ into (4.2), we have

$$
L(h)-A(h)=0
$$

that is equation (3.2).

Expanding the function $h$ in the power series in $p$, we obtain

$$
h(x, y, p)=h_{0}(x, y)+h_{1}(x, y) p+h_{2}(x, y) p^{2}+h_{3}(x, y) p^{3}+\ldots
$$

and the solution to (3.2) is expressed as

$$
T_{f}(x, y)=\lim _{p \rightarrow 1} h(x, y, p)=h_{0}(x, y)+h_{1}(x, y)+h_{2}(x, y)+h_{3}(x, y)+\ldots
$$

Finally, we take an approximate solution of (3.2) in form of truncated series (4.5)

$$
T_{f}(x, y)=\sum_{i=0}^{N_{f}} h_{i}(x, y)
$$

The convergence of HPM for partial differential equations was proved in Biazar and Ghazvini (2009) and Turkyilmazoglu (2011). The outcome of computations indicates that satisfactory results can be obtained with three or four terms in series (4.6). The assumptions relating to the initial approximation $u_{0}$ can be weaker. In further calculation, the initial approximation may be an arbitrary function. Substituting (4.4) into (4.2) and comparing coefficients at subsequent powers of $p$ to zero, we obtain a system of equations from which we calculate $h_{0}, h_{1}, h_{2}, \ldots$ sequentially

$$
\begin{aligned}
& L\left(h_{0}\right)-L\left(u_{0}\right)=0 \\
& L\left(h_{1}\right)+L\left(u_{0}\right)-A\left(h_{0}\right)=0 \\
& L\left(h_{2}\right)-A\left(h_{1}\right)=0 \\
& \vdots \\
& L\left(h_{N_{f}}\right)-A\left(h_{N_{f}-1}\right)=0
\end{aligned}
$$

The Trefftz method is used to determine functions $h_{n}(x, y), n=0,1, \ldots, N_{f}$, which are solutions to successive equations in system (4.7). In this case, the solutions $h_{n}(x, y)$ contain two terms: a linear combination of the Trefftz functions $u_{i}(x, y)$ and a particular solution of the $n$-th equation from system (4.7), see Ciałkowski and Frąckowiak (2000), i.e. 


$$
\begin{aligned}
& h_{0}(x, y)=\sum_{i=1}^{N_{0}} c_{i}^{(0)} u_{i}(x, y)+u_{0} \\
& h_{1}(x, y)=\sum_{i=1}^{N_{1}} c_{i}^{(1)} u_{i}(x, y)+L^{-1}\left[A\left(h_{0}\right)\right]-u_{0} \\
& h_{2}(x, y)=\sum_{i=1}^{N_{2}} c_{i}^{(2)} u_{i}(x, y)+L^{-1}\left[A\left(h_{2}\right)\right] \\
& \vdots \\
& h_{n}(x, y)=\sum_{i=1}^{N_{n}} c_{i}^{(n)} u_{i}(x, y)+L^{-1}\left[A\left(h_{n-1}\right)\right]
\end{aligned}
$$

where the functions $u_{0}, L^{-1}\left[A\left(h_{0}\right)\right]-u_{0}, L^{-1}\left[A\left(h_{n}\right)\right]$ are particular solutions to the appropriate equations from system (4.7) and $L^{-1}$ is the inverse of operator $L$. Since the differential operators $L$ and $A$ are linear, one can rewrite recursive formula (4.8) in a more concise form

$$
\begin{aligned}
& h_{0}(x, y)=\sum_{i=1}^{N_{0}} c_{i}^{(0)} u_{i}(x, y)+u_{0} \\
& h_{n}(x, y)=\sum_{s=0}^{n} \sum_{i=1}^{N_{s}} c_{i}^{(s)} L^{-(n-s)}\left[A^{n-s}\left(u_{i}(x, y)\right)\right]+L^{-n}\left[A^{n}\left(u_{0}\right)\right]-L^{-n+1}\left[A^{n-1}\left(u_{0}\right)\right] \\
& \quad n=1,2, \ldots, N_{f}
\end{aligned}
$$

where $L^{-n}=L^{-1}\left(L^{-(n-1)}\right)$ and $A^{n}=A\left(A^{n-1}\right)$. The description of the use of inverse operators in the Trefftz method can be found in Ciałkowski and Frąckowiak (2000), Grysa et al. (2012).

Known boundary conditions (3.3)-(3.5) help determination of the coefficients of linear combination (4.8) by minimizing the corresponding error functional appropriate for each function $h_{n}(x, y), n=0,1, \ldots, N_{f}$. Sequential determination of the functions $h_{n}(x, y)$ requires each time taking into account the computed functions $h_{n}(x, y)$ in boundary conditions (3.3)-(3.5) after appropriate modification (Grysa and Maciag, 2013). The approximate liquid temperature computed from (4.6) satisfies approximately both equation (3.2) and boundary conditions (3.3)-(3.5).

\section{Results}

Numerical calculations for cooling liquid FC-72 have been performed based on the experimental results described in detail in Piasecka $(2013,2014)$ concerning a forced flow of FC-72 through an asymmetrically heated minichannel, Fig. 3. The flow structures and the void fraction have also been observed. In further calculations, the local void fraction determined at lengths $0.09 \mathrm{~m}$, $0.133 \mathrm{~m}, 0.27 \mathrm{~m}$, and $0.34 \mathrm{~m}$ is approximated with a quadratic function, Fig. 3 .

Approximate temperature distributions of the glass, heating foil and liquid have been determined sequentially. In the first instance, the approximate temperatures of the glass $T_{G}$ and the heating foil $T_{F}$ were calculated knowing both the temperature distribution at the foil-glass interface and the heat flux at the foil inside the surface-liquid interface. Fifteen Trefftz functions $u_{i}(x, y)$ for Laplace's equation were adopted for calculations, i.e. $N_{G}=N_{F}=15$. To determine $T_{G}$ and $T_{F}$, we adopted the particular solution to equation (4.1) in the form $\widetilde{u}(x, y)=-0.5 q_{V} \lambda_{F}^{-1} y^{2}$. The distribution of the fluid temperature could be obtained only after determining the glass and the foil temperature. The liquid temperature $T_{f}$ in the minichannel was determined based on (4.6) with the initial approximation $u_{0}=0$ (other forms of the initial 

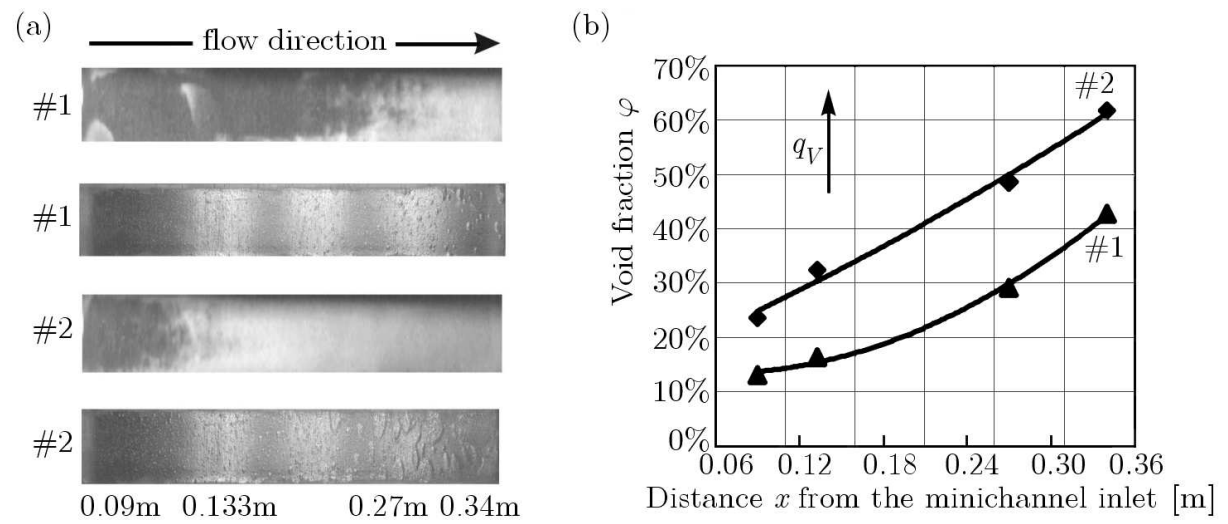

Fig. 3. (a) Hue distribution on the exterior of the minichannel obtained with liquid crystal thermography and the corresponding flow structures observed for the given temperature distribution,

(b) void fraction. Experimental parameters of the runs (Piasecka, 2014); foil parameters: $\delta_{F}=1.02 \cdot 10^{-4} \mathrm{~m}, H=0.35 \mathrm{~m}, \lambda_{F}=8.3 \mathrm{~W} /(\mathrm{mK})$; glass parameters: $\delta_{G}=0.006 \mathrm{~m}, \lambda_{G}=0.71 \mathrm{~W} /(\mathrm{mK})$; for \#1: $G=282 \mathrm{~kg} /\left(\mathrm{m}^{2} \mathrm{~s}\right), \operatorname{Re}=944, p_{\text {in }}=129 \mathrm{kPa}, T_{\text {in }}=293 \mathrm{~K}, T_{\text {out }}=319 \mathrm{~K}, q_{V}=1.92 \cdot 10^{5} \mathrm{~kW} / \mathrm{m}^{3}$; for $\# 2: G=277 \mathrm{~kg} /\left(\mathrm{m}^{2} \mathrm{~s}\right), \operatorname{Re}=009, p_{\text {in }}=139 \mathrm{kPa}, T_{\text {in }}=293 \mathrm{~K}, T_{\text {out }}=334 \mathrm{~K}, q_{V}=2.99 \cdot 10^{5} \mathrm{~kW} / \mathrm{m}^{3}$

approximation $u_{0}$, for example, a harmonic function, did not affect the final result). The following quantities were used in calculations: local void fraction, pressure drop, liquid temperature at the inlet and outlet of the minichannel, liquid saturation temperature and the foil temperature gradient in the foil-liquid contact area along the channel. Three steps of recursion were made approximating $h_{n}(x, y)$ with five Trefftz functions $u_{i}(x, y)$ in each step, i.e. $N_{f}=2$ and $N_{n}=5$. Figure 4 presents two-dimensional temperature distribution of the glass, the foil and the flowing liquid. Application of Trefftz method allowed to obtain two-dimensional temperature distributions in the three neighbouring domains.

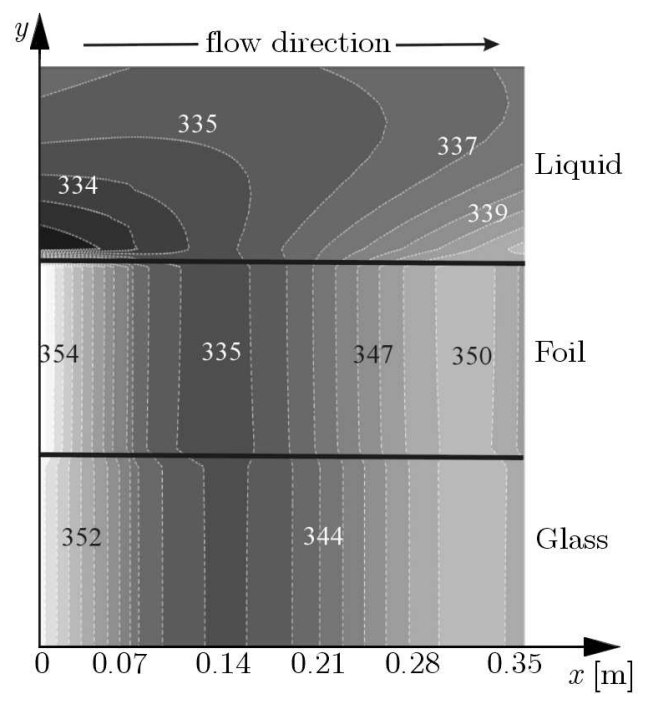

Fig. 4. Temperature of the glass pane and the heating foil determined by the Trefftz method.

Temperature of the liquid obtained HPM / Trefftz method. Additional data: as in Fig. 3 for \#2

To verify the solution obtained by the HPM combined with the Trefftz method, equation (3.2) has been solved using a different approach with an additional simplification. The liquid temperature change along the whole minichannel length has been replaced with the formula from (Bohdal, 2000)

$$
\frac{\partial T_{f}}{\partial x}=\frac{D q}{S G c_{p, f}}
$$


Substituting (5.1) into (3.2), we obtain the Poisson equation. The solution to this equation is given in the form of the sum of the linear combination of the Trefftz functions $u_{i}(x, y)$ and the particular solution

$$
T_{F}(x, y)=\sum_{j=1}^{M_{f}} d_{j} u_{j}(x, y)+\frac{D q \rho_{f}}{S G \lambda_{f}} L^{-1}[w(y)]
$$

The coefficients $d_{j}$ are calculated in the same way as in the Trefftz method. Five Trefftz functions $u_{i}(x, y)$ are taken, analogously to the combination of the HPM with the Trefftz method.

Figure 5 compares the cooling liquid temperature distribution derived from the solution obtained from formulas (4.6) and (5.2). Both approaches produce very similar results in the thermal layer, Fig. 5. This is the result of the fact that the determination of the liquid temperature in the minichannel leads to the solution of the inverse problem.

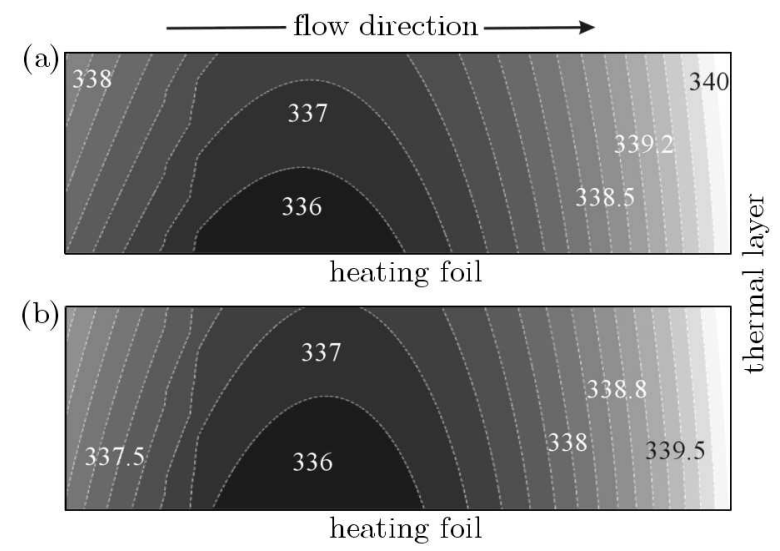

Fig. 5. Temperature of the liquid: (a) obtained by formula (4.6), (b) obtained by formula (5.2),

(c) temperature scale; additional data: as in Fig. 3 for \#2

For correct interpretation of the temperature distribution shown in Fig. 5, one has to take into account the physics of flow boiling. In the considered case, the heat received from the foil is transferred by the bubbles of gas to the center (axis) of the minichannel which can be seen in Fig. 4. For that reason, once the boiling incipience has taken place, the bubbles lower the temperature of the fluid in the immediate neighbourhood of the foil and the temperature of the foil alone, see Fig. 5. The measured temperature of the heating foil at the foil-glass contact, see Fig. 3, confirms that such a phenomenon is observed (i.e. temperature rise and then a rapid drop after the boiling incipience), see Fig. 4 and Fig. 5 as well as the figures presented in Piasecka $(2013,2014)$.

In Hożejowska and Piasecka (2014) and Hożejowska et al. (2014) in order to determine the temperature of the liquid for equation (3.2), the Trefftz functions were derived assuming the velocity $w(y)$ to be a roof or parabolic function. When the velocity profile has a more complex form, liquid temperature can be calculated from the HPM combined with the Trefftz method.

The known temperature field of the liquid is employed to determine the heat transfer coefficient at the contact point of the foil and liquid, calculated from Robin condition (3.9). Figure 6 presents the heat transfer coefficient calculated from Eq. (3.9) when the liquid temperature is calculated using the HPM plus Trefftz method and when the liquid temperature is obtained by (5.2). Concentrating only on the thermal sublayer, we obtain similar plots of heat transfer coefficients, Fig. 6, with differences that do not exceed $5 \mathrm{~kW} /\left(\mathrm{m}^{2} \mathrm{~K}\right)$ on average. A fast increase in the heat transfer coefficient values results from the phase change which accompanies heat transfer. It is observed that when the heat flux supplied to the heating wall grows, the heat transfer coefficient grows too. A further increase in the heat flux results in an increase in the 
void fraction and a decrease in the heat transfer coefficient, Fig. 6. In the enhanced boiling region, heat transfer coefficient values decrease with the distance from the minichannel inlet and with the increasing vapour fraction in the flowing mixture.
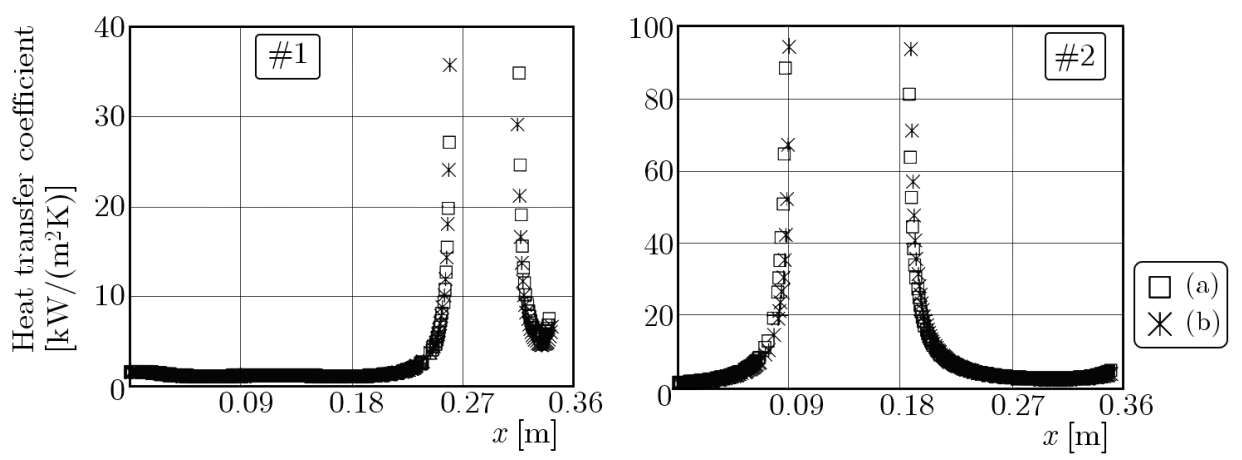

Fig. 6. Heat transfer coefficients as a function of the minichannel length obtained using: (a) HPM plus Trefftz method, (b) Trefftz method and inverse operations; additional data: as in Fig. 3

\section{Conclusions}

The presented combination of the HPM and the Trefftz method helps determination of the approximate two-dimensional temperature distribution of the boiling liquid. The Trefftz method is used to solve the direct heat transfer problem for the glass pane and the inverse problem in the heating foil, determining their temperatures and gradients. Known temperature distributions in the foil-liquid contact area are used to compute the heat transfer coefficient from the Robin boundary condition. The results are summarized and compared with those obtained from the simplified model. The resulting two-dimensional liquid temperature distributions are similar, in particular when the considerations are limited to the thermal layer. An analogous relationship is observed for the heat transfer coefficient calculated for both models. The advantage of the HPM/Trefftz method combination is its simplicity and a small number of steps of recursion to produce a satisfactory result. The number of Trefftz functions used in calculations is also small. In addition, this combination of methods can be used to solve problems described by non-linear equations. Thus, further work will be directed towards determination of the temperature of the liquid phase, vapour phase and the mixture of these two phases in two-phase flows for more complex models than those presented here.

\section{Acknowledgments}

The research has been financially supported by the National Scientific Center from funds granted by virtue of decision No. DEC-2013/09/B/ST8/02825.

\section{References}

1. Al-Khatib M.J., MaciąG A., Pawińska A., 2014, The Trefftz functions in different methods of solving the direct and inverse problems for beam with variable stiffness, 8th International Conference on Inverse Problems in Engineering, 12-15 May, Cracow

2. Biazar J., Ghazvini H., 2009, Convergence of the homotopy perturbation method for partial differential equations, Nonlinear Analysis: Real World Applications, 10, 2633-2640

3. Bohdal T., 2000, Modeling the process of bubble boiling on flows, Archives of Thermodynamics, 21, 34-75 
4. Cialkowski M.J., Frąckowiak A., 2000, Heat Functions and Their Application to Solving Heat Conduction in Mechanical Problems (in Polish), Politechnika Poznańska

5. Grysa K., MaciąG A., 2013, Homotopy perturbation method and Trefftz functions in the source function identification, APCOM\&ISCM, 11-14 December, Singapore

6. Grysa K., MaciąG A., Pawińska A., 2012, Solving nonlinear direct and inverse problems of stationary heat transfer by using Trefftz functions, International Journal of Heat and Mass Transfer, 55, $7336-7340$

7. He J.-H., 1999, Homotopy perturbation technique, Computer Methods in Applied Mechanics and Engineering, 178, 3/4, 257-262

8. HE J.-H., 2000, A coupling method of homotopy technique and perturbation technique for nonlinear problems, International Journal of Non-Linear Mechanics, 35, 37-43

9. He J.-H., 2006, Some asymptotic methods for strongly nonlinear equations, International Journal of Modern Physics B, 20, 10, 1141-1199

10. Herrera I., 2000, Trefftz method: a general theory, Numerical Methods for Partial Differential Equations, 16, 561-580

11. Hetmaniok E., Nowak I., SŁota D., WituŁa R., 2012, Application of the homotopy perturbation method for the solution of inverse heat conduction problem, International Communications in Heat and Mass Transfer, 39, 30-35

12. Hożejowska S., Kaniowski R., Poniewski M.E., 2014, Application of adjustment calculus to the Trefftz method for calculating temperature field of the boiling liquid flowing in a minichannel, International Journal of Numerical Methods for Heat and Fluid Flow, 24, 811-824

13. Hożejowska S., Piasecka M., 2014, Equalizing calculus in Trefftz method for solving two-dimensional temperature field of FC-72 flowing along the minichannel, Heat and Mass Transfer, 50, 8, 1053-1063

14. Hożejowska S., Piasecka M., Poniewski M.E., 2009, Boiling heat transfer in vertical minichannels. Liquid crystal experiments and numerical investigations, International Journal Thermal Sciences, 48, 1049-1059

15. Jafari H., Seifi S., 2009, Solving a system of nonlinear fractional partial differential equations using homotopy analysis method, Communications in Nonlinear Science and Numerical Simulation, 14, 5, 1962-1969

16. MaciąG A., 2011, The usage of wave polynomials in solving direct and inverse problems for two-dimensional wave equation, International Journal for Numerical Methods in Biomedical Engineering, 27, 1107-1125

17. Momani S., Odibat Z., 2007, Comparison between the homotopy perturbation method and the variational iteration method for linear fractional partial differential equations, Computers and $M a-$ thematics with Applications, 54, 7/8, 910-919

18. Piasecka M., 2013, Heat transfer mechanism, pressure drop and flow patterns during FC-72 flow boiling in horizontal and vertical minichannels with enhanced walls, International Journal of Heat and Mass Transfer, 66, 472-488

19. Piasecka M., 2014, Flow Boiling on Enhanced Surfaces of Minichannels (in Polish), Politechnika Świętokrzyska

20. Piasecka M., Poniewski M.E., Hożejowska S., 2004, Experimental evaluation of flow boiling incipience of subcooled fluid in a narrow channel, International Journal Heat and Fluid Flow, 25, 159-172

21. Rajabi A., Ganji D.D., Taherian H., 2007, Application of homotopy perturbation method in nonlinear heat conduction and convection equations, Physics Letters A, 360, 570-573

22. SŁota D., 2011, Homotopy perturbation method for solving the two-phase inverse Stefan problem, Numerical Heat Transfer, Part A, 59, 755-768 
23. Treffrz E., 1926, Ein Gegenstück zum Ritzschen Verfahren, 2 Int. Kongress für Technische Mechanik, 131-137

24. Turkyilmazoglu M., 2011, Convergence of the homotopy perturbation method, International Journal of Nonlinear Sciences and Numerical Simulation, 12, 9-14

25. Zieliński A.P., 1995, On trial functions applied in the generalized Trefftz method, Advances in Engineering Software, 24, 147-155

Manuscript received November 28, 2014; accepted for print May 21, 2015 